\title{
Some Pelvic Limb Imaging of Domestic Cats: Correlation between Computed Tomography and Cross-Sectional Anatomy
}

\author{
Abdel-Mohsen BA, Daghash SM, Sary RG and Farag FM \\ Department of Anatomy and Embryology, Faculty of Veterinary, Medicine Cairo University, Giza, Egypt \\ *Corresponding author: basmarady96@hotmail.com
}

Article History: $21-400 \quad$ Received: 12-Sep-21
ABSTRA C T
The purposes of this study were to describe the anatomical structures of the pelvic limb of the cat by using the cross-
sectional anatomy (CSA) and computed tomography (CT) scan. In this study, we used six adult domestic cats of both
sexes. Identified the different anatomical structures of the pelvic limb in the frozen, fixed sections. The study created
seventeen cross-sections in the pelvic limb of the cat with the concurrent CT images. The sections aimed to demonstrate
the limb's bones, muscles, and tendons and focused on the regional differences. The sections included the thigh, shank
(leg), and metatarsal regions. Each region was sectioned on a proximal, middle, and distal level. The selected slices
were three in the thigh, five in the stifle joint, four in the leg (shank), two through the tarsus, and lastly, three in the
metatarsus (foot). The CT images were compared with the corresponding sections at the same levels, selected for their
identity, and photographed. The relevant anatomical structures were spotted on the transverse slices and labeled similar
structures on the matching CT images.

Key words: Cat, CT scan, Cross-sectional anatomy, Pelvic limb.

\section{INTRODUCTION}

Recently, cross-sectional imaging has become more important in veterinary medicine, to aid exact diagnosis of pathology and surgical planning (Hagen 2012). Learning cross-sectional anatomy (CSA) enables anatomy students to understand the relative position of bodily organs, thereby understanding the three-dimensional architecture. This is an essential learning skill that helps them to interpret radiological images (CT or MRI) in a clinical setting (Jastrow and Vollrath 2003) which undoubtedly, has got an increasing role in modern anatomy education (Heptonstall et al. 2016). However, CT and MRI images are not the best beginning tools to learn reading CSA, as those images do not reflect the natural colors of the body tissues. Students often use color photographic atlases, prosected body crosssections (Samarakoon et al. 2016). The use of computed tomography (CT) in veterinary practice has been increasing rapidly in line with reduced cost, improved availability and the increase in expertise and technology. The uses of CT technology in veterinary research, especially in relation to bone, vasculature and soft tissues are explored and compared in relation to the different species (Keane et al. 2017).

CSA and CT of the pelvic limb of the domestic cat is necessary as part of the diagnosis of injuries of this region.
The ability to obtain cross-sectional images is valuable because it allows inspection of the internal structures and anatomy that cannot be seen on conventional radiographs due to superimposition. The cross-sectional images created during a CT scan can be applied in numerous planes and produce three-dimensional images which can be examined on a computer monitor, published on film, or transmitted to electronic media.

Our current research focuses on the pelvic limb musculature as the region of interest for two main reasons. First is to show the students the complexity of pelvic limb layers which is difficult to integrate from available topographic anatomic references. Second is to present a normal reference to veterinary pathologists and surgeons to facilitate identification of lesions within the bones and musculature of the pelvic limb of the domestic cat. Consequently, CT scans can provide more detailed information related to injuries and/or diseases of the bone and muscles, that helps surgeon in diagnosis of injuries preceded surgical interference.

\section{MATERIALS AND METHODS}

This study was conducted following the ethical principles for animal experimentation adopted by the Institutional

Cite This Article as: Abdel-Mohsen BA, Daghash SM, Sary RG and Farag FM, 2022. Some pelvic limb imaging of domestic cats: correlation between computed tomography and cross-sectional anatomy. International Journal of Veterinary Science 11(2): 229-235. https://doi.org/10.47278/journal.ijvs/2021.101 
Animal Care and Use Committee tracked in the Faculty of Veterinary Medicine Cairo University (Vet. CU. IACUC) VetCU24112020256. Six mature domestic cats obtained from private veterinary clinics in Giza, Egypt with average weights of $3.5-4.5 \mathrm{~kg}$. with indication of euthanasia due to disease unrelated to the pelvic limb were used in this study. The animals were euthanized using IV10mg/kg Diazepam. After euthanasia of four animals, two were fixed by fixative solution (formalin $200 \mathrm{~mL}$, glycerin $50 \mathrm{~mL}$, Dettol $50 \mathrm{~g}$, thymol $50 \mathrm{~g}$, potassium acetate $50 \mathrm{~g}$, alcohol up to $1000 \mathrm{~mL}$ ) injected in arterial system through carotid artery and used for manual dissection of the pelvic limbs to identify the muscles and tendons. The two other animals were frozen at $-15^{\circ} \mathrm{C}$ and then the pelvic limbs were removed and sectioned transversely using a band saw at regular intervals about one centimeter but in tarsal joint sections were made every $0.5 \mathrm{~cm}$. The frozen sections were studied twice, first freshly and then after being fixed in 10\% formalin. Each gross anatomic slice was photographed for later comparison to CT image.

The remaining two specimens were used alive before euthanasia for CT scan. It restrained in customized restraining frames and anesthetized by intramuscular injection of $22 \mathrm{mg} / \mathrm{kg}$ ketamine. The cats were then supported in lateral recumbency with the pelvic limb extended caudally. Computed tomography was performed at 130 kilovolts and $80 \mathrm{~mA}$ by using Hitachi- hyperdense well-defined on CT images CXR 4 Multi-Slice CT Scanner. Continuous transverse series of CT scan of the pelvic limb were obtained every $5-6 \mathrm{~mm}$. The images were printed by using Hitachi-digital printer and photographed. The CT images were matched with the corresponding sections at the same levels and selected for their identity. The relevant anatomical structures were spotted on the transverse slices and labeled with the similar structures on the matching CT images. The nomenclature used was adopted according to the (NAV 2017).

\section{RESULTS}

Seventeen cross sections photographed and matched with their identical corresponding CT images were selected and labeled. The sections were applied at the level of the thigh, stifle, leg, tarsus, and metatarsus. The transverse CT images provided good representation and details of anatomical structures when compared to their identical photography. Particularly, identifiable anatomical structures were labeled on the sections with their analogous corresponding CT images. Bones were the densest tissue on CT images and appeared as the whitest hyperdense tissue, with a well-defined dark shaded medulla. Muscles and tendons appeared on various grey scales on CT images.

Cross section and CT image (Fig. 1/I \& II) at the level of the proximal thigh showed centrally located the femur with well-defined medulla as dark zone in the CT image. Cranial muscles of the thigh were represented in the transverse CT image by massive quadriceps femoris (18). The four heads of the later muscle, the M. rectus femoris (3) which appeared covered with strong fascial investment, M. vastus medialis (4), and the much thicker M. vastus lateralis (5) as well as the M. vastus intermedius (6) could be identified in the anatomical cross section. The caudal thigh, the hamstring muscles were also capacious and were represented by the $M$. semitendinosus (10), M. Semimembranosus (11), M. Biceps femoris (12) and M. caudofemoralis (13). Medial thigh muscles were represented by massive adductor (9) and M. pectineus (7), thin gracilis (8), while the sartorius muscle split into cranial and caudal parts $\left(1 / 1^{\prime} \& 1^{\prime \prime}\right)$. the ischiatic nerve (37) was observed between the biceps femoris and semimembranosus muscles.

Cross section at the level of the middle (Fig. 1/ III \& IV) and distal (Fig. 1/ V \& VI) showed little difference. The femur shifted cranially and consequently the cranial muscles of the thigh, the quadriceps, shrink in mass, and the while on the contrary the caudal thigh muscles were much more massive. The M. gracilis (8) appeared much thicker.

Cross section and CT image (Fig. 2/ I \& II) in the stifle joint cutting through the base of patella $(F)$ articulating with the shallow femoral trochlea (B). The Origin of Both heads of the of M. gastrocnemius (14/14`\& 14“) was observed just caudal to the femur. The M. quadriceps femoris was represented only by the tendon of insertion of the $M$. vastus medialis (4). Medial thigh muscles, the two parts of $\mathrm{M}$. sartorius (1) conjoined to single mass, M. gracilis (8) formed first layer while the M. adductor (9) formed second layer. The caudal thigh muscles predominate the slice and were represented by the semitendinosus (10), $\mathrm{M}$. semimembranosus (11) and M. biceps femoris (12). Between the latter two present the terminal part of the $\mathrm{N}$. ischiadicus (37) caudal to which located a quadrilateral mass of fat, the Corpus adiposum popliteum (35) which appeared as dark mass in the CT image. Cross section and CT image (Fig. 2/III \& IV) in the stifle joint passing through the sesamoids of the gastrocnemius muscle, the medial and lateral fabellae $(\mathrm{G})$ were clearly defined in both cross anatomical and CT scan image. The slice showed no significant difference from the previous one rather than the commence of the M. flexor digitorum superficialis (15). Between the two heads of the of M. gastrocnemius (14/ 14 \& 14 “').

Cross section and CT image (Fig. 2/ V \&VI) in the stifle joint passing through the femoral condyles showed nearly similar configuration to the preceding slice in addition to the appearance of the Cyamella or the popliteal sesamoid bone (S) on the caudolateral aspect of the lateral condyle embedded in the tendon of origin of the Popliteus muscle in both anatomical slice and CT image. The femoral condyles (C and D) and the intercodyloid fossa (E), where the cruciate ligaments, cranial $(\mathrm{Q})$ and caudal $(\mathrm{R})$ could be also identified. The tibial nerve (38) could be identified along the caudal margin of $\mathrm{M}$. gastrocnemius while the fibular nerve (39) between the lateral head of gastrocnemius and biceps femoris. Next slice in the stifle at proximal end of tibia and fibula (Fig. 2/ VII \&VIII) showed the patellar ligament (U) caudal to which a mass of fat, the Corpus adiposum infrapatellare (36) and was observed in the CT image as small dark mass. The popliteal lymph node (16) appeared embedded in the popliteal adipose body.

Cross section and CT image (Fig. 2/ IX \& X) in the stifle joint $\mathrm{j}$ passing through the insertion of the patellar ligament $(\mathrm{U})$ showed the cranially placed tibia $(\mathrm{H})$ which appeared triangular with cranially pointed subcutaneous tibial crest. The fibula (I) acquired lateral situation and 

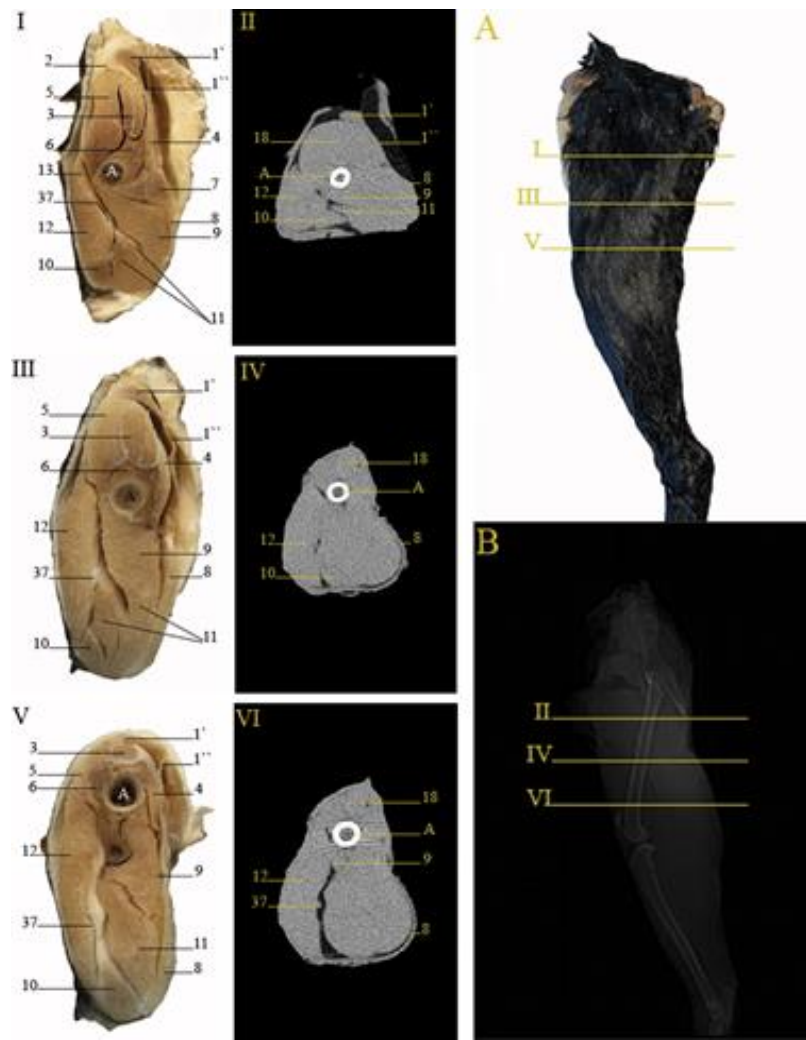

Fig. 1: A and B are photographs showing the selected levels of cross sections and their corresponding computed tomography level in thigh region of left pelvic limb. cross section (I) and Transverse CT image (II) at the level of proximal thigh region 2 $\mathrm{cm}$. distal to the greater trochanter of the femur. cross section (III) and Transverse CT image (IV) at the middle of the thigh, $4.5 \mathrm{~cm}$. distal to the greater trochanter of the femur. cross section (V) and Transverse CT image (VI) at the distal thigh, $0.5 \mathrm{~cm}$. proximal to the base of patella.

could be easily palpated. The M. tibialis cranialis (17) occupied craniolateral position lodged within the extensor groove between the tibial tuberosity and lateral condyle of the tibia. Caudal muscles of the shank were represented by the M. flexor halluces longus (20) which lodged in contact with the caudal surface of the fibula. M. popliteus (25) was small muscle situated just caudal to the tibia, related caudally to the $\mathrm{M}$. flexor digitorum superficialis (15) which was accompanied with the tibial nerve (38) and both were sandwiched between the two heads of gastrocnemius muscle. From the previous slices continued the sartorius (1), gracilis (8) and semimembranosus (11) muscles medially and biceps femoris (12) laterally and the Corpus adiposum popliteum (35) caudally.

Cross sections showed that the muscles of the leg (shank) were arranged around the shank bones into four groups: cranial, lateral, caudal. The cranial group comprised the $\mathrm{M}$. tibialis cranialis, $\mathrm{M}$. extensor digitorum longus. The lateral group constituted the M. peroneus longus and $\mathrm{M}$. extensor digitorum lateralis. The caudal set was further distinguished into deep and superficial muscles. The caudal deep muscles were the M. tibialis caudalis, $\mathrm{M}$. flexor halluces longus, $\mathrm{M}$. flexor digitorum longus and M. popliteus were in direct contact with the caudal surface of the tibia, while the caudal superficial group was formed mainly by Calf muscles that comprised the M. gastrocnemius, M. soleus and M. plantaris. The intermuscular septa between these groups were feeble and mostly loose connective tissue

Cross section and CT image (Fig. 3/ I \& II) the proximal leg region, $1 \mathrm{~cm}$. distal to tibial tuberosity showed the commences of $\mathrm{M}$. extensor digitorum longus (21), M. peroneus longus (23) and M. extensor digitorum lateralis (22) with the craniolateral muscles and the M. soleus. (26) with the caudal shank muscles. Continued to the middle of the leg (Fig. 3/ III \& IV) a similar configuration was noticed in addition to the commences of the $\mathrm{M}$. flexor digitorum longus (19) and $\mathrm{M}$. tibialis caudalis (27) along the caudomedial aspect of the tibia as well as the $\mathrm{M}$. extensor digitorum lateralis (22), laterally.
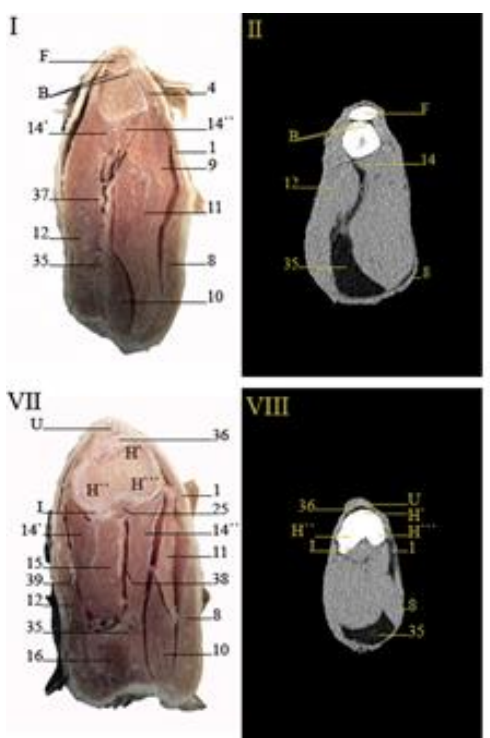

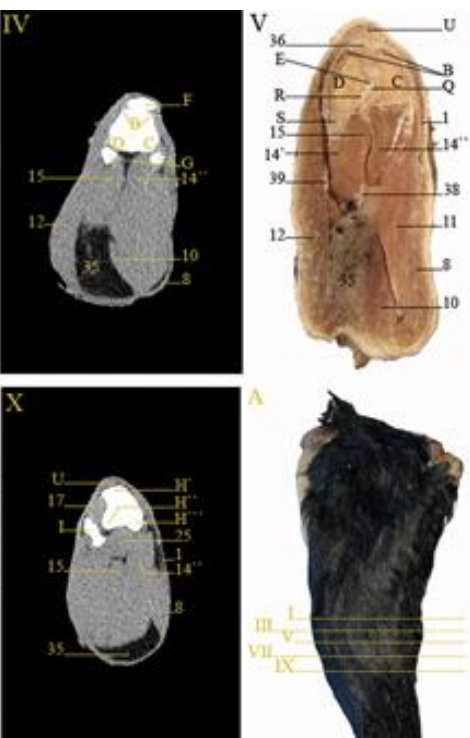

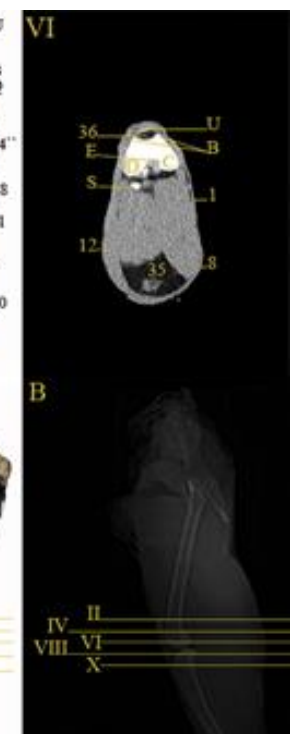

Fig. 2: A and B are photographs showing the selected levels of cross sections and their corresponding computed tomography level in the stifle joint of left pelvic limb. cross section (I) and Transverse CT image (II) through the stifle joint at the level of the base of patella. cross section (III) and Transverse CT image (IV) through the stifle passing through the sesamoids of the M. gastrocnemius. cross section (V) and Transverse CT image (VI) through the stifle joint passing through the femoral condyles at the level of popliteal sesamoid. cross section (VII) and Transverse CT image (VIII) in the stifle joint at the proximal extremity of tibia and fibula. cross section (IX) and Transverse CT image (X) in the stifle joint passing through the insertion of the patellar ligament. 

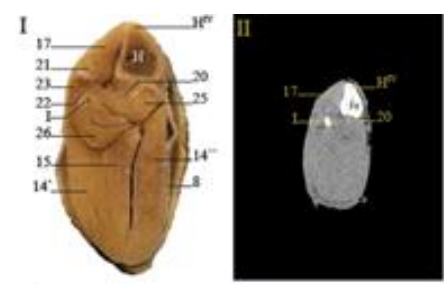

III

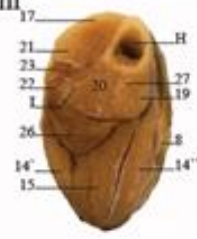

V

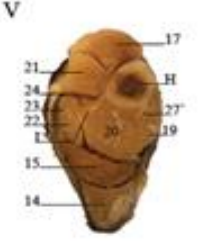

VII

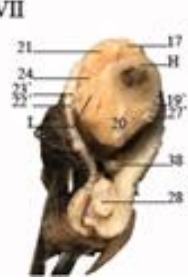

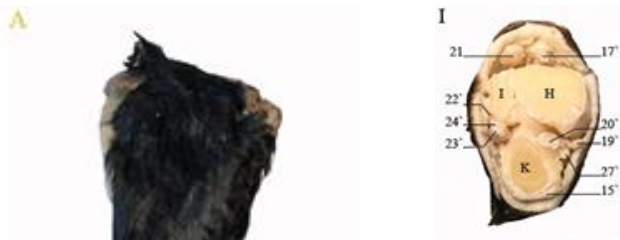

III



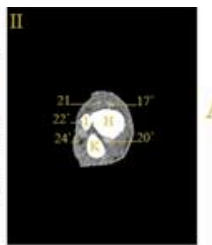

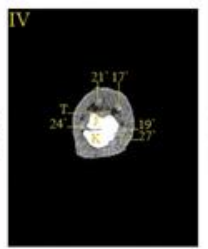

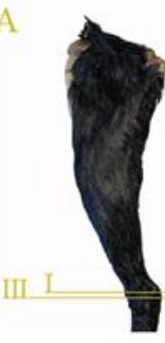

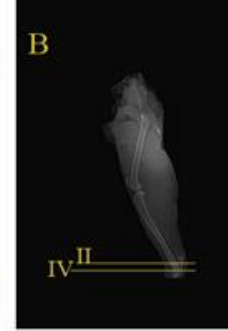

Fig. 4: A and B are photographs showing the selected levels of cross sections and their corresponding computed tomography level in the tarsal joint of left pelvic limb. cross section (I) and Transverse CT image (II) at the level of the distal end of the tibia, fibula, and calcaneus. cross section (III) and Transverse CT image (IV) through the tarsus at the level of the talus and calcaneus.
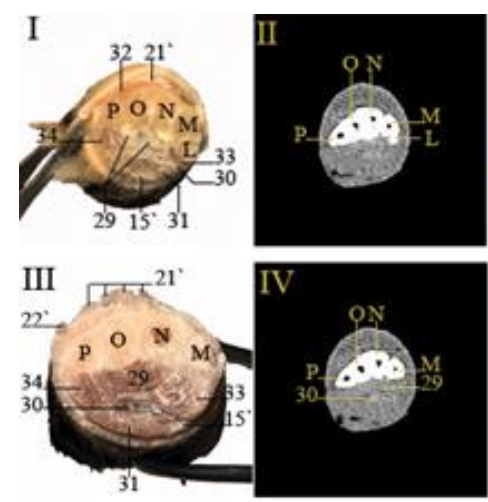
cross sections and their corresponding computed tomography level in the shank(leg) region of left pelvic limb. cross section (I) and Transverse CT image (II) on a level with the proximal leg region, distal to tibial tuberosity by $1 \mathrm{~cm}$. cross section (III) and Transverse CT image (IV) at the level of the middle of the leg region, $3 \mathrm{~cm}$. distal to tibial tuberosity. cross section (V) and Transverse CT image (VI) the level of the distal leg region, $4 \mathrm{~cm}$. proximal to the calcaneus. cross section (VII) and Transverse CT image (VIII) at the level of the distal leg region, proximal to the calcaneus by $2.0 \mathrm{~cm}$.

Cross section and CT image (Fig. 3 / V \& VI) in the distal leg region, $4 \mathrm{~cm}$. proximal to the calcaneus. displayed further discrepancies that comprised the tibia $(\mathrm{H})$ acquired more medial situations, the fibula (I) sinks deeper, the gastrocnemius heads adjoin to crescentic mass (14) that lied in contact with the caudal surface of the superficial digital flexor (15). Moreover, M. peroneus brevis (24) commenced, and tibialis caudalis (27`) tendon replaced the fleshy mass of the muscle.

Cross section and CT image (Fig. 3/ VII \& VIII) through the distal leg region $2.0 \mathrm{~cm}$. proximal to the calcaneus showed that the tibial cross section $(\mathrm{H})$ appeared oval with well-defined dark medulla and the fibula (I) lies on the same transverse axis with the tibia. The section also showed the continuity of M. tibialis cranialis (17) and M. extensor digitorum longus (21) along the craniolateral aspect and M. flexor halluces longus (20) and M. peroneus brevis (24) caudolaterally. It is to add that, the current slice and the succeeding ones showed various tendons of the shank muscles. The major of these tendons is the common calcaneal tendon (28) which appear circular in outline and extended along the caudal aspect of the distal part of the shank and terminal into the calcaneus. Tendo peroneus longus (23`) and Tendo extensor digitorum lateralis (22`)

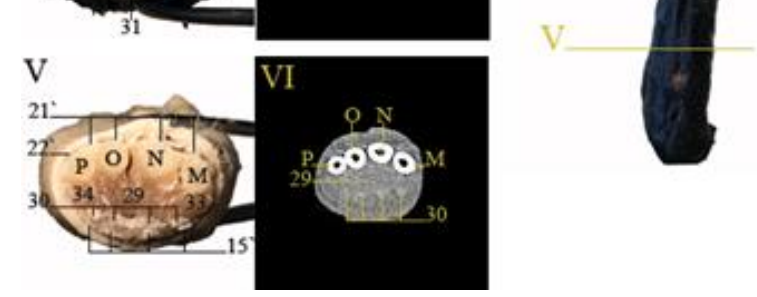

Fig. 5: A is a photograph showing the selected levels of cross sections and their corresponding computed tomography level in the metatarsal region of left pelvic limb. cross section (I) and Transverse CT image (II) on the level of the proximal portion of the metatarsus, $0.5 \mathrm{~cm}$ below the tarsus. cross section (III) and Transverse CT image (IV) on the level of the middle portion of the metatarsus, $4 \mathrm{~cm}$ proximal to the metatarsal pad. cross section (V) and Transverse CT image (VI) on the level of the distal portion of the metatarsus, $0.5 \mathrm{~cm}$ proximal to the metatarsal pad.

were laterally situated while Tendo tibialis caudalis $\left(27^{`}\right)$ and Tendo flexor digitorum longus (19`) located caudomedially. The tibial nerve (38) was observed cranial to the common calcaneal tendon.

Cross section and CT image (Fig. 4/ I \& II) on a level passing through the distal end of the tibia $(\mathrm{H})$, fibula (I), and calcaneus $(\mathrm{K})$ showed the continuity of the tendons of the shank muscles and their relation to the bony elements which could be grouped into four categories, dorsal, plantar, caudolateral, and caudomedial. The dorsal set comprised the M. extensor digitorum longus (21) the only muscle of which the fleshy mass extended to this sectional level in addition to the Tendo tibialis cranialis (17 ). The caudolateral set comprised the Tendo peroneus longus (23`) and Tendo extensor digitorum lateralis (22`) and Tendo 
Int J Vet Sci, 2022, 11(2): 229-235.

Legend for figures from 1-5.
1) M. Sartorius
- 1: pars cranialis
- 1" pars caudalis
2) M. tensor fasciae latae
3) M. rectus femoris
4) M. vastus medialis
5) M.vastus lateralis
6) $\mathrm{M}$. vastus intermedius
7) M.pectineus
8) M.gracilis
9) M.adductor
10) M.semitendinosus
11) M.semimembranosus
12) M.biceps femoris
13) M.caudofemoralis
14) M.gastrocnemius
- 14: caput laterale
- 14": caput mediale
15) M.flexor digitorum superficialis
15) Tendo flexor digitorum superficialis
16) nodi lymphoidei poplitei
17) M.tibialis cranialis
17) Tendo tibialis cranialis
18) M.quadriceps femoris
19) M.flexor digitorum longus
19') Tendo flexor digitorum longus
20) M.flexor halluces longus
20') Tendo flexor halluces longus
21) M.extensor digitorum longus
21') Tendo extensor digitorum longus
22) M.extensor digitorum lateralis
22') Tendo extensor digitorum lateralis
23) M.peroneus longus
23') Tendo peroneus longus
24) M.peroneus brevis
24') Tendo peroneus brevis
25) M. popliteus
26) M.soleus
27) M.tibialis caudalis
27) Tendo tibialis caudalis
28) tendo calcaneus communis
29) $\mathrm{Mm}$. interossei
30) Tendo flexor digitorum profundus
31) M.flexor digitorum brevis
32) M.extensor digitorum brevis
33) M. abductor digiti II
34) M. abductor digiti V
35) corpus adiposum popliteum
36) corpus adiposum infrapatellare
37) N.ischiadicus
38) N. tibialis
39) N.fibularis

peroneus brevis (24) passing between the fibula and calcaneus. At this sectional level the lateral digital extensor tendon was shifted toward the dorsum swapping position with tendon of M. peroneus longus. On the other hand, the caudomedial set of tendons constituted the Tendo flexor halluces longus (20`), Tendo tibialis caudalis (27`) and Tendo flexor digitorum longus (19`) passing within the acute angle made by the tibia and calcaneus. The plantar set was represented by the Tendo flexor digitorum superficialis (15') just caudal to the calcaneus.

Cross section and CT image (Fig. 4/ III \& IV) in the tarsus passing through the talus and calcaneus showed nearly similar configuration of the tendons with the commences of the Tendo extensor digitorum longus (21') which was separated from Tendo tibialis cranialis (17`) by the Trochlea tali (T). The plantar aspect showed the beginning of the Tendo flexor digitorum profundus (30).

Cross section and CT image (Fig. 5/ I \& II) on the level of the proximal portion of the metatarsus, $0.5 \mathrm{~cm}$ below the tarsus showing the $1^{\text {st }}$ rudimentary metatarsal I (L) Ossa metatarsale II (M), Ossa metatarsale III (N), Ossa metatarsale IV (O) and Ossa metatarsale V (P). The metatarsals except the $1^{\text {st }}$ showed well-defined medullary cavity in the CT image. The outline of the metatarsals (IIV) appeared triangular with its base toward the dorsum of the foot, closely adherent with obscure interosseous spaces. Dorsally, the Tendo extensor digitorum longus (21') and Tendo extensor digitorum lateralis (22') were continued overlapping the M. extensor digitorum brevis (32). The plantar aspect of the metatarsal bones showed the $\mathrm{Mm}$. interossei (29). On either side of the later muscle the M. abductor digiti II (33) and M. abductor digiti V (34) were situated. The plantar aspect of the interosseous muscle was overlapped by $\mathrm{M}$. flexor digitorum brevis (31). In between the latter two muscles, the deep digital flexor tendon (30) was positioned while the superficial digital flexor tendon (15') was still undivided and overlap the plantar aspect of the M. flexor digitorum brevis.
Similar configuration was observed in the cross section and CT image in the middle of the metatarsal region (Fig. 5/ III \& IV), $4 \mathrm{~cm}$ proximal to the metatarsal pad with little variances. $1^{\text {st }}$ rudimentary metatarsal I disappeared, the long digital extensor tendons (21') was forked into its digital divisions while the superficial digital flexor tendon broaden and be ready for division. Further progressing to the distal metatarsus (Fig. 5/ V \& VI) the sectional view of the metatarsal bones appeared oval in transverse plan bearing obviously wider medullary cavity and separated by relatively wide interosseous spaces. The furcation of both Tendo extensor digitorum longus (21`) and Tendo flexor digitorum superficialis (15`) was defined on the dorsum and plantar aspect respectively.

\section{DISCUSSION}

The present study correlates anatomic cross-sections with the corresponding CT slices and confirmed the statement made by Farkas (1988) that the cross-sectional anatomy is the foundation for understanding and interpreting the CT-image and with the advent of CT the importance of cross-sectional anatomy cannot be overestimated. On reviewing the available literatures little was found concerning the imaging of the musculoskeletal system of the pelvic limb of the cat. Field and Taylor (2018), Hudson and Hamilton (2010) and Roosa et al. (2020) created an atlas of feline anatomy. Moreover, Samii et al. (1998) discussed the cross-sectional anatomy of the feline thorax and abdomen: comparison of computed tomography and cadaver anatomy while Osorio-Echeverri et al. (2019) designed three-dimensional cat virtual anatomical Software. However, none of the articles documented cross sections or CT scan imaging showing the bones and muscles of the pelvic limb of the domestic cat neither for learning purpose nor demonstrating the anatomical structures of this region. 
Results of our study indicate that the use of anatomic sections is helpful for identifying the bones and muscles that are visible on CT images of the cat pelvic limb. Only nerves and small vessels could not be discerned. Anatomic images made in our study matched closely with corresponding CT and provided detailed views for the normal relevant structures of the bones and muscles. These images could be used as a normal anatomic reference during the diagnosis of the musculoskeletal disorders in this region.

The submission of the cross-sectional anatomy on the pelvic limb of the cat as an approach for anatomical study agreed with Perumal (2018) who employed the sectional anatomy learning tool (SALT) on the spinal levels, thorax, abdomen and pelvis of the human body. Learning crosssectional anatomy (CSA) enables medical students to appreciate the relative position of bodily organs, thereby understanding the three-dimensional architecture of the human body. This is an essential skill that helps them interpret radiological images (CT or MRI) in a clinical setting (De Barros et al. 2001), which undoubtedly, has got an increasing role in anatomy education (Murakami et al. 2014).

The cross sections and CT images in the present study confirm the observation of Mahoney (2012) in cat that the sesamoid bones embedded within the tendon of insertion of the quadriceps femoris (the patella), the medial and lateral heads of the gastrocnemius muscle (medial and lateral fabellae), and the tendon of origin of the popliteal muscle. However, the latter author stated that one or more of these sesamoid bones were not always visible. A statement which could not be recognized in the present study which confirmed that the three sesamoid bones were found in all the examined specimens in both cross anatomic sections and CT images.

Our study proved that considerable amount of infrapatellar fat is present beneath patellar ligament that appeared as a dark mass in corresponding CT image. An observation which was in accordance with Evans and Hermanson (1993) and Robins (1990) who mentioned that the joint capsule is separated from patellar ligament by a huge amount of fat. The current investigation recorded the orientation of the thigh muscles in three groups, cranial, caudal, and medial in relation to the femur. These groups of muscles were designated as cranial, caudal, and medial. The cranial muscles of the thigh were represented by massive quadriceps femoris. The caudal thigh, the hamstring muscles were capacious and were represented by the M. semitendinosus, M. Semimembranosus and M. Biceps femoris. The medial thigh muscles were represented by the adductor, pectineus, gracilis and sartorius muscles. An observation which resembles to a great extent the statement made by Kenneth (2017) and Bordoni and Varacallo (2018) that the human thigh into three fascial compartments: anterior, posterior, and medial. The anterior compartment contained the quadriceps femoris and sartorius, while the posterior compartment, contain the hamstrings while the medial compartment comprises the M. gracilis, M. pectineus, $M$. adductors. The authors added that strong connective tissue fascia formed the septa between the compartments and that the muscles in each of these compartments have the same function, nerve, and blood supply.
Similarly, in accordance with Bordoni and Varacallo (2018) in human, the cross sections in the leg of the cat showed that the muscles of the leg were arranged around the shank bones into three groups: cranial, lateral, caudal. The cranial group comprised the $\mathrm{M}$. tibialis cranialis, $\mathrm{M}$. extensor digitorum longus. The lateral group constituted the M. extensor digitorum lateralis and M. peroneus longus. The caudal set was further distinguished into deep and superficial muscles. The caudal deep muscles were the $\mathrm{M}$. tibialis caudalis, M. flexor halluces longus, M. flexor digitorum longus and $\mathrm{M}$. popliteus, while the caudal superficial group comprised the M. gastrocnemius, M. soleus and M. plantaris.

It is to add that, although the muscle arrangement into three groups in both thigh and leg of the cat was like that in the human as mentioned by Kenneth (2017) and Bordoni and Varacallo (2018) but these groups didn't enclose in separate compartments which might be attributed to the weakness of the intermuscular septa between these groups. A statement which leads as to mention what is called compartment syndrome which is occur in the human thigh and leg and described as a serious condition resulting from increased pressures and muscle damage within any of the three fascial compartments as mentioned by Verwiebe et al. (2009). However, this syndrome was rare and recorded as case report in cat (Montero and Torres 2015) and dog (Gossot et al. 2010).

In agreement with Falcon et al. (2011) the popliteal fat pad in the cat is located between the medial and lateral hamstring muscles and covers the proximal attachments of the gastrocnemius muscles. The arrangement of these muscles forms a pocket caudal to the knee joint, and the fat pad is in this pocket, beneath the crural fascia.

In agreement with Gielen et al. (2001) bony structures in dog including distal end of tibia and fibula, calcaneus and trochlea tali of talus, surrounding tendons could be identified on both cross anatomical sections and CT images, tendons of peroneus longus $\mathrm{M}$. and extensor digitorum lateralis $M$. cross over resulting in changed positions.

Our study was in accordance with El-Ghazali and ElBehery (2018) who mentioned that, the first metatarsal was undeveloped in the cat. It is to add that, the metatarsals except the $1^{\text {st }}$ showed medullary cavity, well-defined in the CT image and appeared wider towards the distal slice of the foot. In this connection Kornmayer et al. (2014) measured the medullary cavity diameter at the mid-diaphysis of the metacarpals and metatarsals in cats, using a statistical software package radiographic and computed tomographic (CT) measurements, to evaluate the clinical relevance of the measurements by examining their relationship to clinically used pin sizes.

\section{Conclusion}

Results of our study indicated that, the use of anatomic sections is helpful for identifying the bones and muscles that are visible on CT images of the cat pelvic limb. Anatomic images made in our study matched closely with corresponding CT and provided detailed views for the normal relevant structures of the bones and muscles. These images could be used as a normal anatomic reference during the diagnosis of the musculoskeletal disorders in this region. Consequently, it could be concluded that the 
cross-sectional anatomy is the basis for understanding and interpreting the CT-image and with the advent of CT the importance of cross-sectional anatomy cannot be overestimated.

Also, the study provided the submission of the crosssectional anatomy (CSA) as learning tool (SALT) on the cat rear limb. Learning cross-sectional anatomy (CSA) enables veterinary anatomy students to appreciate the relative position of bones, joints and surrounding soft structures. This is an essential data that helps them understand radiological images (CT or MRI) in a clinical setting, which clarify the increasing role of anatomical knowledge in presenting good veterinary practitioners.

\section{Author's Contribution}

Farag F.M. and Abdel-Mohsen B.A made the research design and performed the anatomical study with correlation with CT-images. Daghash S.M. helped in photo editing and manuscript revising. Sary R.G helped in practical part of the research and manuscript revising. All authors reviewed and confirmed the last form of manuscript.

\section{REFERENCES}

Bordoni B and Varacallo M, 2018. Anatomy, bony pelvis and lower limb, thigh quadriceps muscle. Stat Pearls Publishing, Treasure Island (FL), USA.

De Barros N, Rodrigues CJ, Rodrigues Jr AJ, De Negri Germano MA and Cerri GG, 2001. The value of teaching sectional anatomy to improve CT scan interpretation. Clinical Anatomy 14: 36-41. https://doi.org/10.1002/10982353(200101)14:1<36::AID-CA1006>3.0.CO;2-G

El-Ghazali HM and El-Behery EI, 2018. Comparative morphological interpretations on the bones of the pelvic limb of New Zealand rabbit (Oryctolagus cuniculus) and domestic cat (Felis domestica). Journal of Advanced Veterinary and Animal Research 5: 410-419. https://doi.org/10.5455/ javar.2018.e292

Evans H and Hermanson J, 1993. The skeleton, arthrology, the muscular system. Miller's Anatomy of the Dog. $3^{\text {rd }}$ Ed. W.B. Saunders, Philadelphia, USA.

Falcon I, Stahl VA and Nichols TR, 2011. Evidence that popliteal fat provides damping during locomotion in the cat. Cells Tissues Organs 193: 336-341. https://doi.org/https://doi.org/ $\underline{10.1159 / 000323680}$

Farkas ML, 1988. Cross-Sectional Anatomy with Corresponding CT Images. Cross-Sectional Anatomy for Computed Tomography. Springer.

Field HE and Taylor ME, 2018. An atlas of cat anatomy, University of Chicago Press.

Gielen IM, De Rycke LM, Van Bree HJ and Simoens PJ 2001. Computed tomography of the tarsal joint in clinically normal dogs. American Journal of Veterinary Research 62: 19111915. https://doi.org/10.2460/ajvr.2001.62.1911

Gossot P, Arnault F, Meynaud P, Palierne S and Autefage A, 2010. Compartment syndrome in a dog. Point Vétérinaire 41: 53-56.

Hagen R, 2012. Cross-sectional imaging: the key to anatomy. Veterinary Record 170: 17-18. https://doi.org/10.1136/vr.e9

Heptonstall N, Ali T and Mankad K, 2016. Integrating Radiology and anatomy teaching in medical education in the UK-the evidence, current trends, and future scope. Academic
Radiology 23: 521-526. https://doi.org/10.1016/j.acra. 2015.12.010

Hudson L and Hamilton W, 2010. Atlas of feline anatomy for veterinarians, Teton New Media.

Jastrow H and Vollrath L, 2003. Teaching and learning gross anatomy using modern electronic media based on the visible human project. Clinical Anatomy 16: 44-54. https://doi.org/10.1002/ca.10062

Keane M, Paul E, Sturrock CJ, Rauch C and Rutland CS, 2017. Computed tomography in veterinary medicine: currently published and tomorrow's vision. Computed TomographyAdvanced Applications. IntechOpen, pp: 271-289. https://doi.org/10.5772/intechopen.68556

Kenneth SS, 2017. Anatomy \& Physiology: The Unity of Form and Function. McGraw-Hill, New York, USA.

Kornmayer, Amort MK, Failing K and Kramer M, 2014. Medullary cavity diameter of metacarpal and metatarsal bones in cats. Veterinary and Comparative Orthopaedics and Traumatology 27: 447-452. https://doi.org/10.3415/VCOT14-01-0014

Mahoney P, 2012. Musculoskeletal Imaging in the Cat: What's normal? What's abnormal? Journal of Feline Medicine and Surgery 14: 13-22. https://doi.org/10.1177/1098612X $\underline{11432823}$

Montero AS and Torres IS, 2015. Síndrome compartimental en un gato. Argos: Informativo Veterinario: 62-64.

Murakami T, Tajika Y, Ueno H, Awata S, Hirasawa S, Sugimoto M, Kominato Y, Tsushima Y, Endo K and Yorifuji H, 2014. An integrated teaching method of gross anatomy and computed tomography radiology. Anatomical Sciences Education 7: 438-439. https://doi.org/10.1002/ase.1430

Nav NAV, 2017. The International Committee on Veterinary Gross Anatomical Nomenclature. Published by the Editorial Committee Hannover (Germany), Columbia, MO (USA), 6th Ed. (Revised version).

Osorio-Echeverri JS, Orrego-Metaute DA, Murillo-Escobar JP and Tamayo-Arango L, 2019. Three-dimensional cat virtual anatomy: development of an interactive virtual anatomical software. Journal of Morphological Sciences 36: 105-114. https://doi.org/10.1055/s-0039-1683964

Perumal V, 2018. A sectional anatomy learning tool for medical students: development and user-usage analytics. Surgical and Radiologic Anatomy 40: 1293-1300 https://doi.org/10.1007/s00276-018-2082-5

Robins G, 1990. The canine stifle joint. 693-760. Canine Orthopedics. $2^{\text {nd }}$ Ed. Lea and Febiger, Philadelphia.

Roosa K, Swislosky E, Caffrey A, Carson J, Chumpitazi C, Conroy K, Epps J, Gray M, Jackson S and Machiarelli S 2020. Atlas of Comparative Anatomy.

Samarakoon L, Vithoosan S, Kokulan S, Dissanayake M, Anthony D, Dissanayake V and Jayasekara R, 2016 Anatomy of teaching anatomy: do prosected cross sections improve students understanding of spatial and radiological anatomy? Anatomy Research International 2016. https://doi.org/10.1155/2016/8984704.

Samii VF, Biller DS and Koblik PD, 1998. Normal crosssectional anatomy of the feline thorax and abdomen: comparison of computed tomography and cadaver anatomy. Veterinary Radiology and Ultrasound 39: 504-511. https://doi.org/10.1111/j.1740-8261.1998.tb01640.x

Verwiebe EG, Kanlic EM, Saller J and Abdelgawad A, 2009. Thigh compartment syndrome, presentation and complications. Bosnian Journal of Basic Medical Sciences 9: S28. https://doi.org/10.17305/bjbms.2009.2751 